\title{
EFEITOS DO ISOLAMENTO SOCIAL PROVOCADO PELA PANDEMIA NA FUNCIONALIDADE DE PESSOAS COM DOENÇA DE PARKINSON
}

Juliana Paulino Dantas da Silva; UFPE/PPGERO - Universidade Federal de Pernambuco/ Programa de Pós Graduação em Gerontologia; juliana.paulino@ufpe.br;

Jaqueline Severo dos Santos; UFPE - Universidade Federal de Pernambuco; jaqueline.ssantos@ufpe.br;

Cleysiane Araújo de Oliveira; UFPE - Universidade Federal de Pernambuco; cleysiane.araujo@ufpe.br;

Kássia Maria Clemente da Silva; UFPE - Universidade Federal de Pernambuco; kassia.maria@ufpe.br;

Nadja Maria Jorge Asano; UFPE - Universidade Federal de Pernambuco; nadja.asano@ufpe.br;

Carla Cabral dos Santos Accioly Lins; UFPE - Universidade Federal de Pernambuco; carla.santos@ufpe.br;

Maria das Graças Wanderley de Sales Coriolano; UFPE - Universidade Federal de Pernambuco; mariagracas.coriolano@ufpe.br

\section{RESUMO}

Introdução: $\mathrm{O}$ isolamento social pode promover prejuízos na funcionalidade, especialmente da população idosa com doenças crônicas. Objetivo: Analisar os efeitos do isolamento social provocado pela pandemia da covid-19 na funcionalidade de pessoas com doença de Parkinson (DP). Métodos: Estudo transversal desenvolvido pelo Programa Pró-Parkinson, realizado por meio da ferramenta google meeting. Foi realizado o teste senta levanta cinco vezes (TSLCV), cujo ponto de corte para redução da funcionalidade e risco de quedas na DP $>16$ segundos; comportamento sedentário verificado pela pergunta: quantas horas por dia você fica envolvido em atividades sentado durante a semana? Cujo ponto de corte $>4$ horas/5dias da semana e percepção de piora em atividades cotidianas durante a pandemia. Os dados foram analisados com o software BioEstat, considerado $\mathrm{p}<0,05$. Resultados: Amostra composta por 20 sujeitos, 10 homens e 10 mulheres, com média de idade $65( \pm 8)$, variando entre 52 a 76 anos, sendo 14 idosos (idade $\geq 60$ anos) e 6 adultos (idade $>50$ anos). Nove sujeitos (45\%) apresentaram tempo superior a 16 segundos para realização do TSLCV, sendo identificada diferença significativa para realização deste teste (média: $12,1 \pm 1,8$ vs $19,2 \pm 2,9$ segundos; $\mathrm{p}<0,0001 *)$. Sete sujeitos (35\%) apresentaram comportamento sedentário, sendo identificada diferença significativa (média: $3 \pm 1$ vs $7 \pm 7$ horas; $p=0,0003^{*}$ ). Atividades em que os sujeitos perceberam piora durante a pandemia foram $\mathrm{n}(\%)$ : 17 (85\%) freezing, $15(75 \%)$ marcha, $12(60 \%)$ girar no leito, $11(55 \%)$ tremor e $5(25 \%)$ sofreram queda durante a pandemia. Conclusão: A pandemia da covid-19 promoveu efeitos negativos na funcionalidade de pessoas com doença de Parkinson.

Palavras- chave: Envelhecimento, Doença de Parkinson, Coronavírus, Funcionalidade. 